\title{
O papel do enfermeiro no manejo ao paciente terminal em UTI: uma revisão integrativa
}

O objetivo deste trabalho é Identificar qual é o papel do enfermeiro no cuidado paliativo de pacientes terminais em Unidade de Terapia Intensiva. Trata-se de uma pesquisa de revisão integrativa, onde os artigos selecionados constituíram compreender o papel do enfermeiro diante ao paciente em cuidado paliativo na Unidade de Terapia Intensiva. Os artigos selecionados foram publicados no período de 2016 a 2021. Foi utilizada a Biblioteca Virtual de Saúde, através das bases de dados: Literatura Latino-Americana e do Caribe em Ciências da Saúde (LILACS); Base de Dados de Enfermagem (BDENF) e Sistema Online de Busca e Análise de Literatura Médica (MEDLINE), a partir dos descritores: Unidade de Terapia Intensiva; Cuidados de Enfermagem e Cuidados Paliativos. A pesquisa demonstrou dificuldades identificadas pelo enfermeiro, os fatores que direcionam a capacitação do profissional sobre tal situação apresentados: inexperiência profissional; lidar com o sofrimento do paciente e da família; falta de trabalho colaborativo entre a equipe; envolvimento dos enfermeiros na tomada de decisões no final da vida. Com base nos dados analisados, observou-se que o fato de lidar com a morte é algo frequente na vida dos profissionais que trabalham na Unidade de Terapia Intensiva, e mesmo assim torna-se um grande desafio para o profissional no dia a dia no manejo desta situação.

Palavras-chave: Unidade de Terapia Intensiva; Cuidados de Enfermagem; Cuidados Paliativos.

\section{The role of the nurses in the management of the therminal patient in the ICU: an integrative review}

The Objective is to identify the role of nurses in palliative care for terminal patients in the Intensive Care Unit. This is an integrative review research, where the selected articles constituted the understanding of the role of nurses in relation to patients in palliative care in the Intensive Care Unit. The selected articles were published from 2016 to 2021. The Virtual Health Library was used, through the following databases: Latin American and Caribbean Literature on Health Sciences (LILACS); Nursing Database (BDENF) and Online Medical Literature Search and Analysis System (MEDLINE), from the descriptors: Intensive Care Unit; Nursing Care and Palliative Care. The research showed difficulties identified by the nurse, the factors that guide the professional training on this situation, presented: professional inexperience; dealing with the suffering of the patient and family; lack of collaborative work among the team; involvement of nurses in decisionmaking at the end of life. Based on the analyzed data, it was observed that the fact of dealing with death is something frequent in the lives of professionals working in the Intensive Care Unit, and even so, it becomes a great challenge for the professional on a daily basis in handling this situation.

Keywords: Intensive Care Unit; Nursing Care; Palliative Care.

Topic: Enfermagem Geral

Reviewed anonymously in the process of blind peer.

Ebraim Kelvin Floriano de Oliveira (iD

Universidade de Sorocaba, Brasil

http://lattes.cnpq.br/7739599698126726

http://orcid.org/0000-0002-1836-3315

braimkel@hotmal.com

Bianca Aparecida Rodrigues Simões (10)

Universidade de Sorocaba, Brasil

http://lattes.cnpq.br/3557294059876028

http://orcid.org/0000-0002-1600-9750

rodrigues.bianca1996@gmail.com

Yngred Moreira Vieira de Souza (iD

Universidade de Sorocaba, Brasil

http://lattes.cnpq.br/8977070325273808

http://orcid.org/0000-0001-5048-8313

yngredsouza@outlook.com.br
Received: 08/06/2021

Approved: 06/07/2021

\author{
Nayara Fernandes (ii \\ Universidade de Sorocaba, Brasi \\ http://orcid.org/0000-0002-6151-4801 \\ nanafernandes27@gmail.com \\ Débora Cabral Nunes Polaz (iD) \\ Universidade de Sorocaba, Brasil \\ http://lattes.cnpq.br/9342411050092970 \\ http://orcid.org/0000-0003-4800-672X \\ debora.polaz@gmail.com \\ Leandro Aparecido Souza \\ Universidade de Sorocaba, Brasil \\ http://lattes.cnpq.br/6090315154831086 \\ http://orcid.org/0000-0001-8828-9918 \\ leandroapsouza14@gmail.com
}

\section{Referencing this:}

OLIVEIRA, E. K. F.; SIMÕES, B. A. R.; SOUZA, Y. M. V.; FERNANDES, N.; POLAZ, D. C. N.; SOUZA, L. A.. O papel do enfermeiro no manejo ao paciente terminal em UTI: uma revisão integrativa. Scire Salutis, v.11, n.3, p.6-13, 2021. DOI: http://doi.org/10.6008/CBPC22369600.2021 .003 .0002 


\section{INTRODUÇÃO}

Através do avanço tecnológico, e o surgimento de novas técnicas e terapias ao decorrer dos anos, proporcionou um maior controle na quantificação e qualificação do tratamento aos mais diversos tipos de doenças, tendo um maior controle em diversos aspectos biológicos apresentados pelo paciente, pois, a maioria dos óbitos ocorre no hospital, sendo o maior número na Unidade de Terapia Intensiva (UTI). Dessa forma o enfermeiro assume um papel primordial na previsão e provisão de recursos necessários ao cuidado, bem como a avaliação das demandas de cada paciente, planejando e implementando ações que permitam ao indivíduo passar pela terminalidade sem sofrimento (PIRES et al., 2020).

Os cuidados paliativos (CP) são ações que atribuem o cuidado muito além da doença em si, constitui-se de uma assistência destinada a melhorar o conforto e a qualidade de vida do paciente e seus familiares. Os pacientes paliativos são aqueles que se encontram em uma condição que ameaça sua continuidade de vida, visto que, neste contexto englobam-se não somente os pacientes em terminalidade de vida, como também aqueles que possuem qualquer doença grave, portanto, essa assistência é fundamental para o controle dos sintomas, alivio da dor e sofrimento, independente do estágio ou prognóstico, sendo de grande qualidade quando fornecido junto à terapia curativa restaurativa. Esta forma de cuidado deve envolver todos da equipe multiprofissional, atuantes na UTI e junto ao familiar para identificar os procedimentos desnecessários e estabelecer ações paliativas necessárias (LOPES et al., 2020).

Os cuidados desses pacientes em terminalidade de vida se fazem muito complexo e difícil para o profissional de enfermagem, principalmente quando há a criação de vínculos entre o profissional e o paciente e a família, nesta situação, onde o mesmo encontra-se fora de possibilidades terapêuticas de cura, cabendo ao enfermeiro junto à equipe multidisciplinar fornecer a prestação do $\mathrm{CP}$, para uma melhor qualidade no tempo restante de vida do paciente, proporcionando alivio da dor, conforto e autonomia através de mecanismos para lidar com as demais possíveis adversidades, também preparando a família na aceitação da possibilidade de morte e prevenção de um luto complicado (CAVALCANTI et al., 2018).

Para a realização dos cuidados paliativos na UTI é importante estabelecer a comunicação entre os profissionais de saúde e com os familiares do paciente, já que a comunicação é um dos pilares da medicina e enfermagem paliativa. A falta de comunicação pode resultar em grandes problemas como a omissão de informações, falta de clareza, contextualização errada, entendimento de informação incorreta. Um bom diálogo para a comunicação de uma má notícia, aos familiares e ao paciente, facilita a compreensão e o entendimento da situação, sendo importante escolher um momento adequado; avaliar o emocional e psicológico do paciente; preparar o paciente e o familiar dizendo ter um assunto difícil para discutir; usar uma linguagem simples e clara; expressar empatia pela dor do paciente; atentar-se como o paciente e os familiares ficaram ao receber a notícia (GULINI et al., 2018).

É notável que na UTI o sentimento negativo de tristeza está perante a assistência ao paciente sem a possibilidade terapêutica e pode causar o adoecimento dos familiares do paciente ou até mesmo do profissional que está prestando a assistência. O sentimento de compaixão está presente para a realização da assistência e pode servir como ensinamento para novas formar de sentir e se envolver com o paciente 
em finitude, é evidenciado que a compaixão está ligada com as objeções do que concerne os cuidados paliativos. Quando todas as medidas terapêuticas são efetuadas, a aceitação da família frente à perda, é retratada de forma mais amena (SANTOS et al., 2018). O lidar com a morte apresenta-se como algo frequente na vida dos profissionais que trabalham em UTI, diante a isto se pode observar, que, alguns profissionais possuem grande dificuldade para encará-la, pois este momento não é somente a falência física, é também uma relação de perda, tanto para a família quanto para a sociedade em geral (SILVEIRA et al., 2016).

Neste contexto, o enfermeiro se mostra fundamental nesse processo, sendo extremamente importante que aconteça o envolvimento emocional e a empatia, desta forma, o presente artigo visa demonstrar, através de uma revisão integrativa da literatura, qual é o papel do enfermeiro no cuidado paliativo de pacientes terminais em unidade de terapia intensiva.

\section{METODOLOGIA}

Trata-se de uma pesquisa de revisão integrativa da literatura, dos quais os artigos que constituíram o estudo abordaram compreender o papel do enfermeiro diante ao paciente em terminalidade de vida, e o cuidado paliativo. Para a busca dos artigos, foi utilizada, a Biblioteca Virtual de Saúde, através das bases de dados: Literatura Latino-Americana e do Caribe em Ciências da Saúde (LILACS); Base de Dados de Enfermagem (BDENF) e Sistema Online de Busca e Análise de Literatura Médica (MEDLINE), a partir dos descritores: Unidade de Terapia Intensiva; Cuidados de Enfermagem e Cuidados Paliativos.

$\mathrm{Na}$ pesquisa foram incluídos artigos originais, que responderam aos objetivos do estudo, publicados entre o período de 2016 a 2021, no idioma português. O presente estudo teve como ênfase a seguinte pergunta norteadora: "Qual o papel do enfermeiro frente ao paciente em Cuidados Paliativos na Unidade de Terapia Intensiva?", onde através da mesma foram atribuídos os critérios de seleção e exclusão do estudo.

Os critérios de exclusão foram definidos a partir dos estudos que não contemplavam ao objetivo da pesquisa, dissertações ou teses, trabalhos incompletos e os que foram publicados anteriormente a 2016. A busca resultou em 220 artigos levantados, dos quais, após análise, foram descartados 209, que não atendiam aos critérios de inclusão, sendo selecionados 11 artigos. A coleta dos dados ocorreu entre março e abril de 2021, e para a consolidação do estudo seguiu-se as etapas: escolha do tema, levantamento bibliográfico preliminar, formulação do problema, elaboração do plano provisório do assunto, busca de fontes, leitura do material, fichamento, organização lógica do assunto e pôr fim a redação do texto.

A análise ocorreu a partir da realização de leitura sistemática dos artigos, onde foram retiradas as ideias centrais de cada estudo, sendo os resultados apresentados, em forma de quadro. Foram analisadas 5 categorias dos artigos: Título, ano de publicação, autores, objetivo e resultados.

\section{RESULTADOS E DISCUSSÃO}

Os 11 artigos selecionados e analisados neste estudo estão apresentados no quadro a seguir: 


\begin{tabular}{|c|c|c|c|c|}
\hline Título do estudo & $\begin{array}{c}\text { Ano de } \\
\text { Publicação }\end{array}$ & Autores & Objetivo & Resultados \\
\hline $\begin{array}{l}\text { Conforto no final de vida na } \\
\text { terapia intensiva: percepção } \\
\text { da equipe multiprofissional }\end{array}$ & 2020 & (PIRES et al.) & $\begin{array}{l}\text { Analisar a percepção da equipe } \\
\text { multiprofissional sobre o conforto no } \\
\text { final de vida na terapia intensiva }\end{array}$ & $\begin{array}{l}\text { Foi um estudo realizado com } 50 \text { profissionais da equipe } \\
\text { da Unidade de terapia Intensiva de um hospital e foi } \\
\text { utilizado um questionário sociodemográfico e uma } \\
\text { semiestrutura para a coleta de dados. Foram } \\
\text { entrevistados médicos, enfermeiros, fisioterapeutas, } \\
\text { técnicos de enfermagem, nutricionistas, assistentes } \\
\text { sociais e psicólogo. } \\
\text { Os profissionais entrevistados, relataram que é } \\
\text { indispensável promover o conforto físico e psicológico } \\
\text { no ambiente da UTI em pacientes terminais. }\end{array}$ \\
\hline $\begin{array}{l}\text { Vivências de Enfermeiros no } \\
\text { Cuidado às Pessoas em } \\
\text { Processo de Finitude }\end{array}$ & 2020 & (LOPES et al.) & $\begin{array}{l}\text { Conhecer e explorar as vivencias } \\
\text { emocionais pregressas dos enfermeiros } \\
\text { perante a finitude/morte e o processo } \\
\text { de morrer em cuidados intensivos. }\end{array}$ & $\begin{array}{l}\text { Os resultados apontaram que a maior parte dos } \\
\text { entrevistados referiu como sentimento negativo à } \\
\text { tristeza diante do paciente em finitude, e como } \\
\text { sentimento positivo compaixão. A principal dificuldade } \\
\text { perante o doente em finitude foi a ausência de } \\
\text { protocolos que definem e dão continuidade ao cuidado } \\
\text { paliativo. O conforto como objetivo para aliviar a dor e } \\
\text { sofrimento foi elencado como principal método para } \\
\text { lidar com paciente em finitude. Constatou-se ainda o } \\
\text { despreparo dos enfermeiros na graduação perante o } \\
\text { processo de morrer. }\end{array}$ \\
\hline $\begin{array}{l}\text { Princípios dos cuidados } \\
\text { paliativos em terapia } \\
\text { intensiva na perspectiva dos } \\
\text { enfermeiros }\end{array}$ & 2018 & $\begin{array}{l}\text { (CAVALCANTI } \\
\text { et al.) }\end{array}$ & $\begin{array}{l}\text { Avaliar a percepção dos enfermeiros } \\
\text { intensivistas acerca da adesão aos } \\
\text { princípios dos cuidados paliativos na sua } \\
\text { prática assistencial }\end{array}$ & $\begin{array}{l}\text { Estudo realizado com um total de } 104 \text { enfermeiros, que } \\
\text { trabalhavam em } 12 \text { diferentes unidades de terapia } \\
\text { intensiva em cinco hospitais. } \\
\text { Os princípios demonstrados de maior relevância } \\
\text { voltados ao cuidado assistencial são: } \\
\text { - Alivio de dor e outros sintomas associados } \\
\text { - } \quad \text { Garantia da qualidade de vida e do morrer } \\
\text { - Priorizar sempre o melhor interesse do } \\
\text { - Raciente } \\
\text { Sespeitar a autonomia do doente e seus } \\
\text { mesmo com uma média elevada são: } \\
\text { - Afirmar a vida e considerar a morte como } \\
\text { - Am processo normal da vida } \\
\text { Avaliar o custo-benefício a cada atitude } \\
\text { médica assumida }\end{array}$ \\
\hline $\begin{array}{l}\text { Fatores preditores de óbito } \\
\text { em Unidade de Terapia } \\
\text { Intensiva: contribuição para } \\
\text { a abordagem paliativista }\end{array}$ & 2018 & (GULINI et al.) & $\begin{array}{l}\text { Identificar preditores de óbito na } \\
\text { Unidade de Terapia Intensiva e } \\
\text { relacionar pacientes elegíveis para } \\
\text { cuidados paliativos. }\end{array}$ & $\begin{array}{l}\text { Pesquisa realizada entre os meses de março a outubro } \\
\text { de 2015, sendo elegíveis para o estudo } 170 \text { pacientes } \\
\text { que permaneceram internados por mais de } 24 \text { horas, } \\
\text { tendo como taxa de mortalidade de } 29 \% \text { destes } \\
\text { pacientes ( } 50 \text { pacientes morreram no hospital), onde } \\
\text { foram classificados em dois grupos G1(pacientes que } \\
\text { evoluíram a óbito: } 35 \text { pacientes na UTI e } 15 \text { pacientes na } \\
\text { enfermaria) e G2(pacientes que tiveram alta e foram } \\
\text { para casa:120 pacientes). } \\
\text { Os pacientes que evoluíram a óbito apresentaram como } \\
\text { característica: } \\
\text { - Pacientes mais velhos; mais frágeis } \\
\text { - Menor funcionalidade e maior tempo de } \\
\text { - Internação } \\
\text { - Falto neurológico agudo e não traumático }\end{array}$ \\
\hline $\begin{array}{l}\text { Análise da Escala de Perroca } \\
\text { em Unidade de Cuidados } \\
\text { Paliativos }\end{array}$ & 2018 & $\begin{array}{l}\text { (SANTOS et } \\
\text { al.) }\end{array}$ & $\begin{array}{l}\text { O Sistema de Classificação de Pacientes } \\
\text { (SCP), é utilizado nos hospitais para } \\
\text { gerenciar o planejamento da } \\
\text { enfermagem. Um desses instrumentos } \\
\text { criados, foi a Escala de Perroca, no qual, } \\
\text { o uso é recomendado pelo COFEN. Esse } \\
\text { instrumento possui treze indicadores } \\
\text { baseados nas necessidades humanas. } \\
\text { Com isso, o objetivo desse estudo era } \\
\text { identificar como os enfermeiros utilizam } \\
\text { a escala de Perroca no cuidado paliativo, } \\
\text { podendo assim, identificar pacientes } \\
\text { com maior necessidade de cuidado e um } \\
\text { possível prognostico. }\end{array}$ & $\begin{array}{l}\text { Para esse estudo, foi analisado através do banco de } \\
\text { dados do Hospital, os pacientes que foram utilizados } \\
\text { essa escala. Foram analisados idade, classificação pela } \\
\text { escala de Perroca, tempo de internação, sexo, data do } \\
\text { óbito ou alta. } \\
\text { O resultado obtido foi que entre } 2008 \text { e } 2016 \text { foram } \\
\text { internados } 2.486 \text { pacientes na Unidade de Cuidados } \\
\text { Paliativos, a mediada de tempo resultou em } 12 \text { dias de } \\
\text { internação. Desses } 2.486 \text { pacientes, } 53,4 \% \text { deles tiram } \\
\text { alta. Desses } 53.4 \% \text {, todos precisaram de cuidados semi- } \\
\text { intensivos ou intensivos. } 46.5 \% \text { foi decretado óbito. } \\
\text { Com isso, concluiu-se que o objetivo do cuidado paliativo } \\
\text { é proporcionar a melhor qualidade de vida ao paciente e } \\
\text { seus familiares, para assim, identificar possíveis } \\
\text { prognósticos e diminuir o número de óbitos. }\end{array}$ \\
\hline $\begin{array}{l}\text { Cuidados Paliativos em } \\
\text { Unidade de Terapia } \\
\text { Intensiva: Percepções dos } \\
\text { Profissionais } \\
\text { Enfermagem }\end{array}$ & 2017 & (FARIA et al) & $\begin{array}{l}\text { Compreender a percepção da equipe } \\
\text { de Enfermagem sobre os cuidados } \\
\text { paliativos a pacientes em estado } \\
\text { terminal. }\end{array}$ & $\begin{array}{l}\text { Foram entrevistados } 15 \text { profissionais de enfermagem } \\
\text { onde } 4 \text { era enfermeiros e } 11 \text { técnicos de enfermagem. } \\
\text { Desses } 15 \text { profissionais } 7 \text { eram homens e oito mulheres } \\
\text { com idade variada de } 24 \text { a } 43 \text { anos. O tempo de atuação } \\
\text { foi usado como variável também com profissionais de } 2 \\
\text { meses a } 20 \text { anos de atuação na enfermagem. Após a } \\
\text { análise das entrevistas foram separadas nas seguintes } \\
\text { categorias: } \\
\text { - a percepção e a vivência da equipe de enfermagem } \\
\text { sobre o cuidado paliativos; } \\
\text { - como o cuidado paliativo é aplicado e aturando junto à } \\
\text { família no enfrentamento do estado terminal. }\end{array}$ \\
\hline
\end{tabular}




\begin{tabular}{|c|c|c|c|c|}
\hline $\begin{array}{l}\text { A equipe da Unidade de } \\
\text { Terapia Intensiva frente ao } \\
\text { cuidado paliativo: discurso } \\
\text { do sujeito coletivo }\end{array}$ & 2017 & (GULINI et al.) & $\begin{array}{l}\text { Conhecer a percepção dos profissionais } \\
\text { de saúde de uma Unidade de Terapia } \\
\text { Intensiva acerca do cuidado paliativo }\end{array}$ & $\begin{array}{l}\text { Estudo realizado com } 37 \text { profissionais da saúde (12 } \\
\text { enfermeiros, } 11 \text { técnicos de enfermagem, cinco } \\
\text { fisioterapeutas e nove médicos). } \\
\text { Abordou-se como as principais ideias centrais extraídas } \\
\text { dos relatos: } \\
\text { - Cuidado na fase terminal da vida sem } \\
\text { - } \text { medidas fúteis. } \\
\text { - Cuidados de conforto } \\
\text { Falta uniformizar a assistência e a } \\
\text { capacitação para a equipe. }\end{array}$ \\
\hline $\begin{array}{l}\text { Sofrimento } \text { Moral dos } \\
\text { Enfermeiros, em Situações } \\
\text { de Final de Vida, em } \\
\text { Unidades de Terapia } \\
\text { Intensiva. }\end{array}$ & 2017 & $\begin{array}{l}\text { (RODRIGUES E } \\
\text { COSTA et al.) }\end{array}$ & $\begin{array}{l}\text { Compreender as práticas exercidas } \\
\text { pelos enfermeiros, na Unidade de } \\
\text { Terapia Intensiva (UTI), em situações de } \\
\text { final de vida, e relacioná-las ao } \\
\text { sofrimento moral. }\end{array}$ & $\begin{array}{l}\text { Estudo realizado diante a análise de } 11 \text { enfermeiros } \\
\text { entrevistador que atuam na UTI de uma instituição de } \\
\text { Minas Gerais. A média de tempo de formação dos } \\
\text { profissionais varia de } 5 \text { a } 11 \text { anos além de possuírem } \\
\text { média de seis anos atuando em Unidade de Terapia } \\
\text { Intensiva. } \\
\text { - } \quad \text { Foram utilizadas as seguintes questões no } \\
\text { - } \quad \text { como são. } \\
\text { vida, ou seja, quando o paciente não } \\
\text { - } \text { Qusponde mais ao tratamento curativo? } \\
\text { Como você percebe sua participação nas } \\
\text { tomadas de decisão nas situações de final } \\
\text { de vida? } \\
\text { Qual o seu sentimento quando a tomada de } \\
\text { decisão vai contra a sua opinião ou contra } \\
\text { seus valores? }\end{array}$ \\
\hline $\begin{array}{l}\text { Ortotanásia nas unidades } \\
\text { de terapia intensiva: } \\
\text { percepção dos enfermeiros } \\
\text { Júlio }\end{array}$ & 2017 & $\begin{array}{l}\text { (SANTANA et } \\
\text { al.) }\end{array}$ & $\begin{array}{l}\text { Esse estudo tem como objetivo } \\
\text { compreender a visão do enfermeiro no } \\
\text { processo de morte e morrer. }\end{array}$ & $\begin{array}{l}\text { A unidade de terapia intensiva é composta por } \\
\text { monitoramento } 24 \text { h por dia com uma imensa tecnologia } \\
\text { dos aparelhos, que monitoram todos os pacientes e } \\
\text { também, proporcionam conforto e bem-estar. } \\
\text { Para esse estudo, foi realizada uma coleta de dados, no } \\
\text { qual doze enfermeiros participaram, no qual, era } \\
\text { necessário ter pelo menos três anos em terapia } \\
\text { intensiva. As questões norteadoras eram sobre o } \\
\text { significado do processo morrer com dignidade } \\
\text { (ortotanásia) e sobre o prolongamento do sofrimento na } \\
\text { terapia intensiva. } \\
\text { Com base nisso, grande parte dos entrevistados } \\
\text { concordaram que era necessário proporcionar o melhor } \\
\text { possível para aquele paciente. Porém, em alguns casos, } \\
\text { percebeu-se que há uma limitação nas condutas } \\
\text { terapêuticas e acreditam que o prolongamento } \\
\text { mecânico, possa causar sofrimento ao paciente. } \\
\text { Percebeu-se que mesmo a enfermagem, sabendo o } \\
\text { processo de nascer e morrer, ainda é difícil a aceitação } \\
\text { dos profissionais, pois, os profissionais querem fazer de } \\
\text { tudo para prolongar a vida do paciente, porém, o } \\
\text { paciente acaba sofrendo e não tendo uma morte } \\
\text { tranquila, como exige a Ortotanásia. } \\
\text { Com isso, concluiu-se que a equipe apresenta dúvidas } \\
\text { sobre o momento certo de cessar as medidas curativas, } \\
\text { e ainda, famílias que não são orientadas corretamente } \\
\text { acaba dificultando todo o processo. Verificou-se também } \\
\text { que é necessária a compreensão de que cada paciente é } \\
\text { único e apresenta suas particularidades, fisiopatologias e } \\
\text { cuidados diferentes, sendo assim, é necessário um bom } \\
\text { relacionamento interpessoal que se deve valorizar a } \\
\text { pessoa humana para contribuir para humanizar o } \\
\text { processo de morte e morrer. }\end{array}$ \\
\hline $\begin{array}{l}\text { Validação de protocolo } \\
\text { assistencial de enfermagem } \\
\text { para pacientes em cuidados } \\
\text { paliativos* }\end{array}$ & 2016 & $\begin{array}{l}\text { (SANTOS } \\
\text { al.) }\end{array}$ & $\begin{array}{l}\text { Descrever todo o processo de validação } \\
\text { do conteúdo do protocolo assistencial } \\
\text { da enfermagem para pacientes que } \\
\text { estão em cuidados paliativos e } \\
\text { internados em Unidade de Terapia } \\
\text { Intensiva. }\end{array}$ & $\begin{array}{l}\text { Esse estudo foi composto em duas fases, a primeira foi a } \\
\text { elaboração do protocolo assistencial e segunda fase foi a } \\
\text { validação de conteúdo, por meio da avaliação do } \\
\text { protocolo. } \\
\text { Alguns itens contemplados foram a identificação, nível } \\
\text { de consciência, ventilação, hidratação, higiene corporal } \\
\text { entre outros. E as intervenções foram divididas nas } \\
\text { dimensões biológicas, psicológicas, social, espiritual e as } \\
\text { intervenções no ato da terminalidade e cuidados pós- } \\
\text { morte, } \\
\text { Onze enfermeiros participaram, e foi realizado um } \\
\text { percentual de concordância nos itens abordados nas } \\
\text { fases e houve grande concordância dos profissionais. } \\
\text { Com isso, o protocolo assistencial mostrou-se válido em } \\
\text { seu conteúdo. }\end{array}$ \\
\hline
\end{tabular}




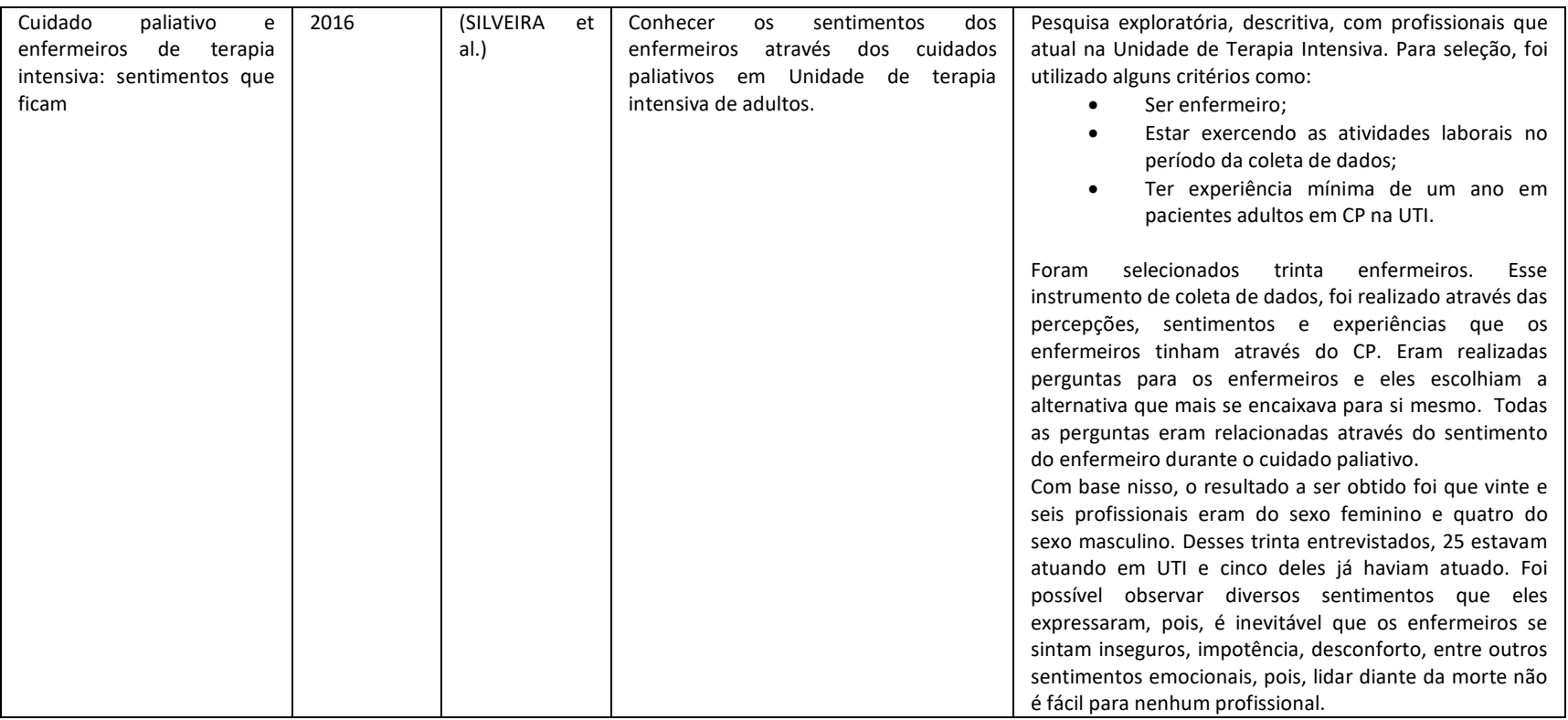

Tanto para Cavalcanti (2018) quanto Gulini (2018), a capacitação e a educação continuada são pilares fundamentais para qualificar a assistência, além de habilidades técnicas, o enfermeiro atua como intermédio entre a equipe e a família. De acordo com apontamentos levantados por Silveira (2016) e Gulini (2017), é fundamental que o enfermeiro tenha o conhecimento de todas as necessidades de seu paciente e o compreenda-o em sua totalidade, por meio de uma comunicação competente entre o profissional, paciente e a família. A fim de aprimorar a assistência e oferecer o suporte necessário.

De acordo com Faria (2017) o enfermeiro deve, qualificar seu cuidado de forma humanizada, flexibilizando-se a partir da necessidade do paciente, através da realização de práticas muita das vezes não realizadas em sua rotina diária.

A assistência do enfermeiro em pacientes sem prognostico de cura, transfere grande carga psicológica, Silveira (2016) e Lopes (2020) retratam o grande impacto emocional, diante a assistência prestado a pacientes em cuidado paliativo. Sentimento de frustação, tristeza, ansiedade e impotência são manifestados. Para Silveira (2016), é indispensável a transparência, do enfermeiro para com o paciente e a família, sobre o prognostico da doença e a possível evolução ao óbito. É retratado por Faria (2017) e Rodrigues et al. (2017), o déficit de conhecimento do enfermeiro, diante a compreensão do cuidado paliativo, representado através disso a fragilidade na formação do profissional.

O "morrer com dignidade" retratado por Santana (2017) e Gulini (2018), manifestam a notoriedade de preservar a autonomia do paciente, controlando sintomas e proporcionando conforto, a fim de que, a morte seja objetiva ao seu tempo certo. Para Lopes (2020) e Pires (2020), o enfermeiro lida constantemente com sentimentos e emoções. O enfermeiro demonstra sentimentos de compaixão, sensibilidade e humanidade, da assistência prestada a esse paciente, sendo um elemento essencial na assistência. É inevitável que ocorra um vínculo emocional entre paciente e seus familiares com a equipe de saúde, o atendimento humanizado e o apoio terapêutico são fundamentais, devido as oscilações de sentimentos que são evidenciados diariamente. A equipe de enfermagem lida constantemente com a finitude e morte. $\mathrm{O}$ sentimento de tristeza é presente, ainda assim, o enfermeiro demanda deixa-lo de lado, 
a fim de fornecer e qualificar o cuidado prestado.

Segundo Santos et al. (2016), a validação do protocolo assistencial de enfermagem para pacientes em cuidados paliativos, foi essencial, pois, era possível obter um seguimento das intervenções a serem seguidas, na qual eram divididas em biológicas, psicológicas, sociais e cuidados realizados no ato da terminalidade ou após a morte, além da identificação, avaliação do nível de consciência, hidratação e higiene corporal.

Sendo assim, Santos (2018) relata a necessidade da utilização da escala de Perroca, pois, é com base nos dados obtidos que há a possibilidade de observar o número de internações, altas e óbitos ocorridos em determinado período, possibilitando e qualificando os cuidados prestados aos pacientes terminais.

\section{CONCLUSÕES}

O estudo evidenciou a importância do enfermeiro frente ao cuidado paliativo em pacientes em unidade de terapia intensiva, sendo esta assistência corriqueiramente vivenciada na unidade de terapia intensiva, onde o cuidado tem como objetivo qualificar a assistência prestada, proporcionando alivio da dor e conforto. A pesquisa demonstrou dificuldades identificadas pelo enfermeiro, frente a assistência a pacientes em cuidados paliativos. Os fatores que direcionam a capacitação do profissional sobre tal situação, apresentados: Inexperiência profissional; lidar com o sofrimento do paciente e da família; Falta de trabalho colaborativo entre a equipe; Envolvimento dos enfermeiros na tomada de decisões no final da vida. Sendo assim faz-se relevante a promoção de estratégias que busquem melhorar o ambiente de trabalho e ressaltar o reconhecimento e valorização do enfermeiro nesse setor. Enfatizando também a importância do diálogo estabelecido entre a equipe multiprofissional de forma a garantir que sua identidade e sua autonomia sejam preservadas.

\section{REFERÊNCIAS}

CAVALCANTI, I. M. C.; OLIVEIRA, L. O.; MACÊDO, L. C.; LEAL M. H. C.; MORIMURA, M. C. R.; GOMES, E. T.. Princípios dos cuidados paliativos em terapia intensiva na perspectiva dos enfermeiros. Rev Cuidarte, v.10, n.1, p.1-10, 2018. DOI: http://dx.doi.org/10.15649/cuidarte.v10i1.555

FARIA, T. N. T.; CARBOGIN, F. C.; ALVES, K. R.; TOLEDO, L. V.; MARQUES, D. A.. Cuidados paliativos em unidade de terapia intensiva: percepções dos profissionais de enfermagem. Revista de enfermagem UFPE on line, v.11, n.5, p.19962002, 2017. DOI: http://dx.doi.org/10.5205/reuol.930281402-1-RV.1105sup201704

GULINI, J. E. H. M. B.; NASCIMENTO, E. R. P.; MORITZ, R. D.; VARGAS, M. A. O.; MATTE, D. L.; CABRAL, R. P.. Fatores preditores de óbito em Unidade de Terapia Intensiva: contribuição para a abordagem paliativista. Revista da Escola de Enfermagem da USP, v.52, p.1-7, 2018. DOI: http://dx.doi.org/10.1590/S1980-220X2017023203342

GULINI, J. E. H. M. B.; NASCIMENTO, E. R. P.; MORITZ, R. D.; ROSA, L. M.; SILVEIRA, N. R.. A equipe da Unidade de Terapia Intensiva frente ao cuidado paliativo: discurso do sujeito coletivo. Revista da Escola de Enfermagem da USP, v.51, n.51, 2017. DOI:

http://dx.doi.org/10.1590/S1980220X2016041703221 LOPES, M. F. G. L.; MELO, Y. S. T.; SANTOS, M. W. C. L.; OLIVEIRA, D. A. L.; MACIEL, A. M. S. B.. Vivências de enfermeiros no cuidado às pessoas em processo de finitude. Revista Ciência Plural, v.6, n.2, p.82-100, 2020.

PIRES, I. B.; MENEZES, T. M. O.; CERQUEIRA, B. B.; ALBUQUERQUE, R. S.; MOURA, H. C. G. B.; FREITAS, R. A.. Conforto no final de vida na terapia intensiva: percepção da equipe multiprofissional. Revista Acta Paulista de Enfermagem, v.33, p.1-7, 2020. DOI: http://dx.doi.org/10.37689/acta-ape/2020A00148

COSTA, M. R.; TEIXEIRA, I.; GUIMARÃES, I. R.; BALIZA, M. F.; BOUSSO, R. S.; POLES, K.. Sofrimento moral dos enfermeiros em situações de final de vida em Unidades de Terapia Intensiva Português/Inglês. Revista de enfermagem UFPE on line, v.11, 2017. DOI:

http://dx.doi.org/10.5205/reuol.10620-94529-1SM.1109sup201714 
SANTANA, J. C. B.; DUTRA, B. S.; CARLOS, J. M. M.; BARROS, J. K. A.. Ortotanásia nas unidades de terapia intensiva: percepção dos enfermeiros. Revista Bioética, v.25, n.1, p.158-670, 2017. DOI: http://dx.doi.org/10.1590/198380422017251177

SANTOS, C. E.; KLUG, D.; CAMPOS, L.; LOSEKANN, M. V.; NUNES, T. S.; CRUZ, R. P.. Análise da Escala de Perroca em Unidade de Cuidados Paliativos. Revista da Escola de Enfermagem da USP, v.52, p.10-40, 2018. DOI: http://dx.doi.org/10.1590/S1980-220X2017037503305
SANTOS, E. C.; OLIVEIRA, I. C. M.; FEIJÃO, A. R.. Validation of a nursing care protocol for patients undergoing palliative care. Revista ACTA Paulista de Enfermagem, v.29, n.4, p.363-730. DOI: http://dx.doi.org/10.1590/19820194201600051

SILVEIRA, N. R.; NASCIMENTO, E. R. P.; ROSA, L. M.; JUNG, W.; MARTINS, S. R.; FONTES, M. S.. Cuidado paliativo e enfermeiros de terapia intensiva: sentimentos que ficam. Revista Brasileira de Enfermagem, v.69, n.6, 2016. DOI: http://dx.doi.org/10.1590/0034-7167-2016-0267

A CBPC - Companhia Brasileira de Produção Científica (CNPJ: 11.221.422/0001-03) detém os direitos materiais desta publicação. Os direitos referem-se à publicação do trabalho em qualquer parte do mundo, incluindo os direitos às renovações, expansões e disseminações da contribuição, bem como outros direitos subsidiários. Todos os trabalhos publicados eletronicamente poderão posteriormente ser publicados em coletâneas impressas sob coordenação da Sustenere Publishing, da Companhia Brasileira de Produção Científica e seus parceiros autorizados. Os (as) autores (as) preservam os direitos autorais, mas não têm permissão para a publicação da contribuição em outro meio, impresso ou digital, em português ou em tradução. 